\title{
Scheduling Scheme Based on Estimated-Delay in Wireless Sensor Network
}

\author{
Zhi Zhang ${ }^{1}$ and Fang $\mathrm{Li}^{2,3, *}$ \\ ${ }^{1}$ Hainan College of Vocation and Technique, Haikou 570216, China \\ ${ }^{2}$ College of Computer Science, Chongqing University, Chongqing 404100, China \\ ${ }^{3}$ Chongqing City Management College, Chongqing 404100, China
}

\begin{abstract}
In the low duty cycle wireless sensor network with unreliable link quality, how to meet designated delay requirements and maximize network lifetime is the focus of this study. This paper proposes the Scheduling Scheme based on Estimated-delay(SSE), which could satisfy decreasing energy consumption as well as delivery delay in the low duty cycle wireless sensor network by utilizing the estimated-delay from the current node to the destination node. We compare our SSE algorithm with other algorithms in the simulation. The results show that SSE could significantly improve the performance in the low duty cycle wireless sensor network compared with other algorithms at the same required delay.
\end{abstract}

Keywords: Link quality, low duty cycle wireless sensor network, node scheduling scheme.

\section{INTRODUCTION}

A critical issue in wireless sensor network is the energy consumption of the sensor nodes for that the sensor nodes are often randomly deployed in a large special area which couldn't have energy supplements. According to the research of current battery storage capacity [1], unless there is a new breakthrough in the material, the battery storage capacity has reached a bottleneck over decades. It's impossible for the researchers to extend the short network lifetime by improving the battery of the node. Low duty cycle wireless sensor network improves the network lifetime, while it also brings new problems which include low link quality as well as long delivery delay. Using Node Scheduling Scheme to overcome the limitations in low duty cycle wireless sensor network has attracted a lot of attention.

This paper studies the Node Scheduling Scheme in wireless sensor network, and then proposes Scheduling Scheme based on Estimated-delay (SSE) under unreliable link quality in low duty cycle wireless sensor network. As we know, in wireless sensor network, nodes energy is major consumed in the transmission work slot [2], with the ability of computing in each node, the node could predict the delivery delay between it and the destination node, then select a path from it to the destination node based on the least delay. During the transfer process, each node could predict new delay based on this path to the destination node and dynamically adjust it's work slot according to it's energy as well as the required delivery delay to the destination node. Based on this, we design the Scheduling Scheme for low duty cycle wireless

*Address correspondence to this author at the Chongqing City Management College, Chongqing 404100, China; Tel: 86-13996206329;

E-mail:241209@qq.com sensor network, to improve the performance of energy consumption under certain required delivery delay, then maximize the network lifetime. The main innovation of this paper is as follows:

1) Considering the certain quality link of the low duty cycle wireless sensor network, optimizing the delivery path which satisfies the requirement delivery delay.

2) Adjusting the work slot based on the remaining energy of the current node as well as the next node, to ensure that each node in the network could consume energy uniformly, thereby improve the whole network lifetime.

The rest of this paper is organized as follows: First of all, we summarize related work in Section 2. In Section 3, we then explain our algorithm. Section 4 evaluates our design. We finally conclude this paper along with future work in Section 5 .

\section{RELATED WORK}

The scheduling scheme algorithm could be divided into two directions, one is determined scheduling scheme, which determines the redundant nodes based on geographic information, then make these nodes to be asleep; the other is random scheduling scheme which is independent of geographic information. The famous algorithm of front, such as SI algorithm [3] proposed by Siqueria et al., reduces the number of work nodes in the premise of ensuring regional coverage, as well as reduces network energy consumption. However, it does not apply to the general sensor network for it is based on clustering and single-hop wireless sensor network. Paul and others proposed the scheduling scheme [4] for dynamic 
clustering wireless sensor network to improve the node energy effectiveness. Its design for the feature of mobile node is based on cluster, so that it makes the redundant nodes to be asleep. However, the algorithm is highly relied on the position of the nodes, not suitable for general low duty cycle wireless sensor network.

The related research about random scheduling scheme is ongoing because it can adapt to the actual environment, such as Randomized Scheduling Scheme (RS) [5], it firstly utilizes the energy model to calculate the energy consumption per node, but it is not suitable for a very wide range use because the calculation process is too simple to adapt to the actual environment. And in the Linear Distance-based Scheduling Scheme (LDS) [6] which is based on linear distance, the calculation process takes into account the distance between nodes, considers that the farther distance brings about the higher sleep probability, and reduces the energy consumption to a large extent. But, it does not ensure a uniform energy consumption of each node, so that some nodes fail obviously earlier than the other, thereby reducing the lifetime of the whole network. Based on the uniform energy consumption, the Balanced-Energy Scheduling Scheme (BS) [7] improved the scheme performance; it can ensure that each node uniform consumes energy, so that each node can work till most of the nodes fail at last, to extend the lifetime of the whole network. However, its design is suitable for high density cluster-based sensor network, couldn't directly use in the low duty cycle network. The well-known random scheduling scheme for the low duty cycle network is TOSS [8], proposed by $\mathrm{Cao}$ et al. It is optimized for detection of rare and urgent events, considers the delay to be composed of detection delay and delivery delay, and proposes optimizations for both. However, it does not consider the conditions under low duty cycle with unreliable link quality between nodes due to low data transmission success ratio [9]. How to improve the delivery ratio as well as node energy consumption based on certain required delivery delay in low duty cycle with unreliable link quality, is the topic of our design.

\section{SSE ALGORITHM}

In the network having $100 \%$ link quality, all the communication between the nodes will be successful, does not need retransmission. Therefore, in the low duty cycle wireless sensor network, if the link quality is $100 \%$, the network delivery delay is the sum of the sleep delay from the source node to the destination node. However, in practical wireless sensor network applications, due to the environmental signals as well as other reasons, the $100 \%$ link quality between the nodes is basically impossible to achieve [10], so the communication failure is unavoidable, and the retransmission brings about too large delivery delay in the actual applications, because the node only wake one time in a cycle (typically tens of seconds or even longer), if the first transmission fails, then the current node must wait for the next cycle of the next hop (the receiving node) to continue the data transmission.

Our SSE algorithm includes the selection of the optimal path as well as the node scheduling scheme, which is based on the estimation of the delivery delay in different paths from the source node to the destination node. SSE selects the path which has the least delivery delay expectation as the optimal path, and the delivery delay is:

$$
E(d(i j))=\min \left[E_{1}(d(i j)), E_{2}(d(i j)) \ldots E_{m}(d(i j))\right]
$$

It is possible that the least delay couldn't satisfy the required delivery delay, so it needs to adjust the work slot of the current node (which has the message to be transmitted) or the next node(the next hop of the current node) during the delivery process, thus considers the required delivery delay as well as the uniform node's energy consumption of the whole network, so that the network lifetime extends. Set the link quality between two neighbor nodes $\mathrm{A}$ and $\mathrm{B}$ is $P_{A B}$, then the success ratio after $n$ retransmissions is:

$$
P(A B)=\sum_{n_{A B}=0}^{n-1} P_{A B}\left(1-P_{A B}\right)^{n_{A B}}
$$

Suppose the maximum number of retransmission for each node is $\mathrm{N}$, the delivery delay between the nodes $\mathrm{i}$ and $\mathrm{j}$ in different path with $k$ hops could be estimated as:

$$
\begin{aligned}
& E[d(i j)]=\sum_{n_{i 1}=0}^{N-1} \ldots \sum_{n_{(k-2)(k-1)}=0 n_{(k-1) j}=0}^{N-1} P_{i 1}^{N-1}\left(1-P_{i 1}\right)^{n_{i 1}} \\
& \ldots P_{(k-1) j}\left(1-P_{(k-1) j}\right)^{n_{(k-1) j}} \times[d(i 1)+\ldots+d((k-1) j) \\
& +T \times\left(n_{i 1}+\ldots+n_{(k-1) j}\right]
\end{aligned}
$$

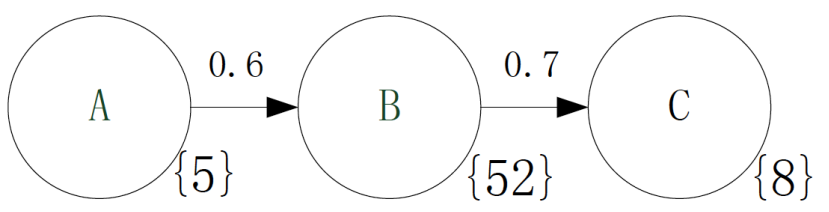

Fig. (1). The model of delivery process under certain link quality.

As shown in Fig. (1), we assume that the duty cycle $\mathrm{T}$ of each node is 100 seconds, the work slot in a cycle of the node $\mathrm{A}$ is $T_{A}=\{5\}$, and the node $\mathrm{B}$ is $T_{B}=\{52\}$, node $\mathrm{C}$ is $T_{C}=\{8\}, P_{i j}$ is the link quality between neighboring nodes $\mathrm{i}$ and $\mathrm{j}, \mathrm{N}$ represents the maximum number of retransmissions in each node, that means the current node discards the packet when the retransmissions is greater than N. In Fig. (1), the link quality between node $\mathrm{A}$ and node $\mathrm{B}$ is 0.6 . Then the delivery delay between the $\mathrm{A}$ and $\mathrm{C}$ as the Equation 4 is estimated as follows:

$$
\begin{aligned}
& E\left(d(A C)=\sum_{n_{A B}=0}^{N-1} \sum_{n_{B C}=0}^{N-1} P_{A B}\left(1-P_{A B}\right)^{n_{A B}} P_{B C}\left(1-P_{B C}\right)^{n_{B C}}\right. \\
& \times\left[d(A B)+T \times n_{A B}+d(B C)+T \times n_{B C}\right]
\end{aligned}
$$

In Equation 4, $\mathrm{n}$ represents the number of retransmissions between nodes. In the low duty cycle wireless sensor network, nodes have randomly different work slots, which mean that the delivery delay between neighboring nodes is large. Our algorithm utilizes the solution to reduce this delay by increasing the work slot in a cycle of a current node.

Consider the two-hop link between nodes $\mathrm{A}$ and $\mathrm{C}$, if the link quality is $100 \%$, the delivery delay is $\mathrm{E}[\mathrm{d}(\mathrm{AC})]=47+$ 
$48+8=103 \mathrm{~s}$. While consider the link quality as (Fig. 1), assume that the maximum number of transmission is 3 (the probability of 3 times retransmission at the link quality 0.7 is $(1-0.7)^{3}=0.027$, that means, the probability of more than 3 times retransmission is already low, so this paper sets the maximum number of retransmissions at 3 , namely $\mathrm{N}=3$, the expectation of the delivery delay between $\mathrm{A}$ and $\mathrm{C}$ is:

$\mathrm{E}[\mathrm{d}(\mathrm{AC})]=43.26+25.58+11.45+34.10+15.27+1.82+20.37$ $+8.12+3.04=163.01 \mathrm{~S}$

The delay increases to 1.5 times, this is only two hops, it will be more large in multi-hop transmission. Therefore, it's necessarily to use the appropriate strategy to improve the performance in unreliable link quality, thereby improving network efficiency.

As shown in Fig. (1), the original $\mathrm{T}$ of node $\mathrm{A}$ and node $B$ is $\{5\}$ and $\{52\}$ respectively, the link quality between $A$ and $\mathrm{B}$ is 0.6 , then the estimated delivery delay from nodes $\mathrm{A}$ to $\mathrm{B}$ is:

$\mathrm{E}[\mathrm{d}(\mathrm{AB})]=0.6 \times 47+0.6 \times 0.4 \times(47+100)+0.6 \times 0.2^{2} \times$ $(47+200)=9.05 \mathrm{~S}$

When the node B increases the work slot $\{15\}$, the duty cycle of node $\mathrm{B}$ becomes $\{15,52\}$, now the new delay is:

$\mathrm{E}[\mathrm{d}(\mathrm{AB})]=0.6 \times 10+0.6 \times 0.4 \times(10+37)+0.6 \times 0.2^{2} \times$

$(10+37+63)=19.92 \mathrm{~S}$

The delay decreases obviously, while nodes increase a work slot but does not significantly increase energy consumption in low duty cycle network. It is obvious that this delay is not the least, the less the new slot interval between the two neighboring nodes, the lower the delivery delay could be achieve; the lowest value is achieved at the interval of 1. For example, in this case, if the node B increases the work slot $\{6\}$, then:

$\mathrm{E}[\mathrm{d}(\mathrm{AB})]=0.6 \times 1+0.6 \times 0.4 \times(1+46)+0.6 \times 0.22 \times(1+$ $46+54)=14.30 \mathrm{~S}$.

However, in practical applications of wireless sensor network, it's difficult to meet the case that the work slot interval between neighboring nodes is 1 . Our algorithm adjusts the work slot of the current node during the delivery process from the source node to the destination node.

It is certain that the source node needs to have the information of the work slot and the link quality of each node along the possible delivery path for predicting the delivery delay. The work slot of each node can be obtained at the network initialization, but the following conditions will dynamically change the node's work slot: 1) the new node of the network; 2) no energy in some nodes; 3) nodes modify their work slot. While link quality between nodes can be obtained only after a few of communication processes and alters by the environment as well as other factors dynamically. Therefore, it is necessary to make all the nodes in the network get the accurate link quality and the work slot dynamically. A simple way to solve the problem is sharing this information with additional communication, but it requires more energy consumption, then decreases the lifetime of the network as well as provides some unnecessary communication interference. This paper proposes a new way to share these information based on the normal communication [11]. All the nodes in the network have their information table that includes the link quality as well as the work slot information and the remaining energy. During the initialization of the network, node $i$ has all the nodes' work slot, and the default value of the link quality based on the statistics data in similar applications, is $P_{a v g}$. After a period of operation, each node has its own link quality from the actual communication; the new link quality is calculated as follow:

$P_{A B}=\alpha \cdot P_{a v g}+\beta \cdot \frac{S_{A B}}{T_{A B}}$

Where $\alpha, \beta$ are adjustment factors, in general, $\alpha=\beta=0.5$. The nodes exchange their information with it's neighbor node during the normal communication process, thereby improving the accuracy of the estimation of the delivery delay. Of course, this process does not guarantee that all nodes in the network have the actual information of each node, especially in the initialization of the network and where network nodes environment as well as other factors change rapidly, it might affect the accuracy of the estimation. But the current node estimated the new delay from the current node to the destination node with its latest information, thereby improving the reliability of the prediction delay values. During the delivery process, the current node may increase its work slot dynamically. If the estimation of the delivery delay calculated by the current node $\mathrm{i}$ to the destination node $\mathrm{j}$, satisfies the required delivery delay, it needn't increase the work slot; the tactics is as follow:

$I_{i}= \begin{cases}E(d(i j)) \leq R(d) & \text { False } \\ E(d(i j))>R(d) & \text { True }\end{cases}$

In which the $R(d)$ is the required delivery delay, if $I_{i}$ is false, node i needn't increase the work slot, Or if the $I_{i}$ is true, the current node compares the remaining energy with the next node, it increases its work slot if the current node has more energy than the next node, that is:

$I_{i}= \begin{cases}E_{\text {remain }}(i) \leq E_{\text {remain }}(i+1) & \text { False } \\ E_{\text {remain }}(i)>E_{\text {remain }}(i+1) & \text { True }\end{cases}$

In which the $E_{\text {remain }}(i)$ is the remaining energy of node $\mathrm{i}$, or else, the current node estimated the new delay $E_{i+1}(d(i j))$ by assuming that the next node increases its work slot, when the new delay satisfies the required delay, the current node needn't increase it's work slot, that is:

$I_{i}= \begin{cases}E_{i+1}(d(i j)) \leq R(d) & \text { False } \\ E_{i+1}(d(i j))>R(d) & \text { True }\end{cases}$

Each node in the network makes decisions based on its information table, after estimation of the delivery delay, increasing the work slot, achieves the reliable packet delivery process. Our whole design is as follow:

\subsection{Estimation of the Delivery Delay}

Each node in the network has the data of the nodes distribution, work slots, link quality as well as the remaining energy after network layout. When one node (source node) 
needs to send data to the destination node, the source node estimates the delivery delay with Equation 4, to determine the delivery path with the Equation 1.

\subsection{Increasing the Work Slot}

Packet delivery goes along the path from the source node to the destination node, the current node receives the packet, then calculates the delay from the current node $i$ to the destination node; with Equation 4. To make decision that whether increasing the work slot, Equations 6-8.

\subsection{Reliable Packet Delivery}

Packet is transmitted sequentially until it reaches the destination node. Each node updates its neighbor nodes' information table during the communication process, then each node has the new situation of the whole network dynamically. Then, it could make the precise estimation as well as the adjustment. And the uniform energy consumption extends the network lifetime. We would testify in section 5 that our SSE could achieve increased lifetime as well as the less delay than others.

\section{SIMULATION}

In this section, we compared SSE with TOSS algorithm under the configuration of Table 1 about the minimum increased work slot and the network lifetime. With the wireless sensor network works, each node has less remaining energy, the network lifetime is over when $30 \%$ of the nodes has no remaining energy. And the minimum increase in work slot is the number of work slot during a delivery process from the source node to the destination node to satisfy the required delay.

Table 1. Simulation configuration.

\begin{tabular}{|c|c|}
\hline Parameters & Description \\
\hline \hline Sensing Range Radius (meters) & 10 \\
\hline The Number of the Nodes & 100 \\
\hline Work Slot Percents in a Cycle & $(1 \% \sim 5 \%)$ The default is $1 \%$ \\
\hline The Time of Each Slot (Seconds) & 1 \\
\hline Cycle of Each Node (Seconds) & 100 \\
\hline Link Quality & $60 \% \sim 90 \%$ \\
\hline Maximum Number of Retransmissions & 20 \\
\hline Energy Consumption in Send Slot & 1 \\
\hline Energy Consumption in Receive Slot & 0.8 \\
\hline Originally Energy in Each Node & 30000 \\
\hline $\begin{array}{c}\text { Average Packet Generation } \\
\text { Interval(Seconds) }\end{array}$ & 1000 \\
\hline
\end{tabular}

As shown in Fig. (2), with the decrease in the required delay, the number of the increasing work slot would decrease in SSE as well as TOSS algorithm. But at the same required delay, SSE has less number than the TOSS. For example, in the case of required delay value 1000 seconds, SSE increases the number of work slot by 2 times, and TOSS by 4 times. With minimum required delay in the network, majority nodes on the path would increase their work slot. When all the nodes on the path have increased their work slot, the performance of SSE algorithm is equivalent to TOSS algorithm. But that is not the normal situation in low duty cycle network. Even if the required delay is 500 seconds, TOSS algorithm increases the minimum number of work slots to 17 , while SSE algorithm increases the number to 15, SSE algorithm still has a higher performance than TOSS. That means, SSE could save more node energy consumption, and then extends the network lifetime. As shown in Fig. (3), as the required delay increases from 500 seconds to 1000 seconds, SSE has $40 \%$ increased network lifetime than TOSS at 1000 seconds, while it has an extended $30 \%$ lifetime than TOSS at 500 seconds.

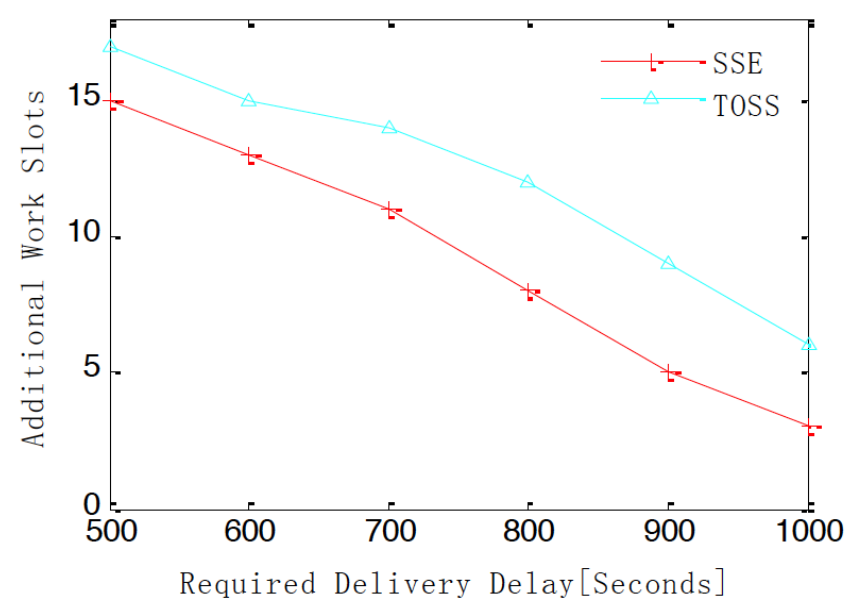

Fig. (2). The additional work slots in different required delivery delay.

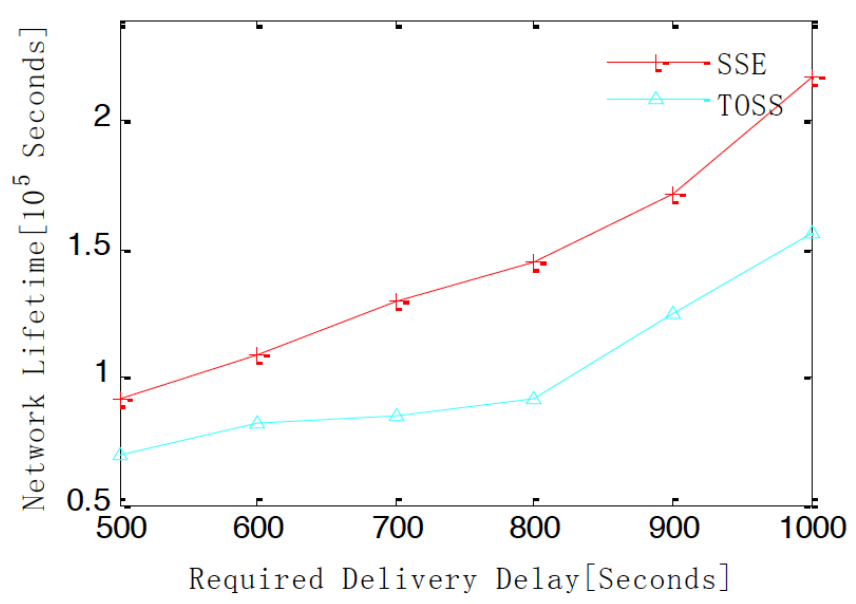

Fig. (3). The network lifetime in different required delivery delay. 
In Fig. 4, when the required delivery delay is $500 \mathrm{sec}-$ onds, with the increase of the nodes' work cycle, the network lifetime is significantly reduced for the node's sleep time turns to be less. And when the duty cycle changes from $1 \%$ to $5 \%$, SSE could extends lifetime more than TOSS, that is nearly $40 \%$ in the best case, while $25 \%$ in the worst case.

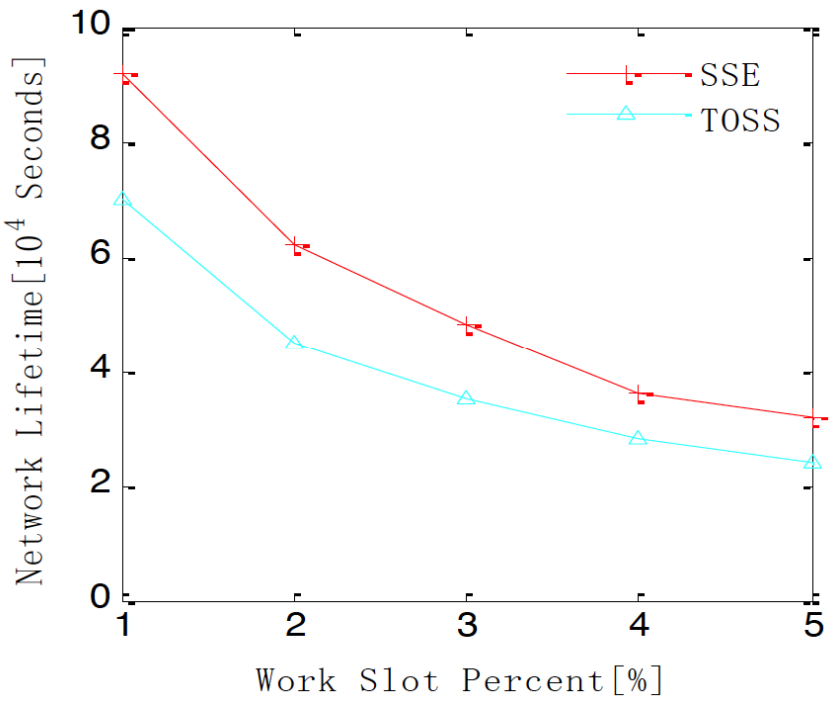

Fig. (4). The network lifetime in different work slot percent.

\section{SUMMARY}

This paper focuses on how to extend the lifetime of the low duty cycle wireless sensor network as well as satisfies the required delivery delay. Simulation results show that our algorithm could extend the network lifetime better than others. But there still have some limitations in our algorithm, such as the estimation of the delay according to the direction from the source node to the destination node, so that from the source node to the destination node the delay could meet the requirement, but from the destination node to the source node in this path the delay is not the optimal. Improving the design to make the delay optimal at the two directions, will give a better performance in the future.

\section{CONFLICT OF INTEREST}

The authors confirm that this article content has no conflict of interest.

\section{ACKNOWLEDGEMENTS}

This work is supported by the Major Research Project of the National Natural Science Foundation of China under Grant No. 90818028, Natural Science Foundation Project of CQ CSTC:2011BB2064.

\section{REFERENCES}

[1] R. Kyoho, T. Goya, W. Mengyan, and T. Senjyu, “ Thermal units commitment with demand response to optimize battery storage capacity," In: Power Electronics and Drive Systems (PEDS), IEEE $10^{\text {th }}$ International Conference, 2013, pp. 1207-1212.

[2] Y. Cao, S. Guo, and T. He, "Robust multi-pipeline scheduling in low-duty-cycle wireless sensor network," In; Proceedings of IEEE Infocom, 2012.

[3] M. Zhang, M.C. Chan, and A.L. Ananda, "Coverage protocol for wireless sensor network using distance estimates," In: R.W. Cruz, and Heinzelman, Eds. Proceeding of the Mesh and Ad Hoc Communications and Network (SECON'07), 2007, pp. 183-192.

[4] S. Paul, and N.K. Sao, "An energy efficient hybrid node scheduling scheme in cluster based wireless sensor network," In: World Congress on Engineering(WCE), 2011.

[5] W. Ye, J. Heidemann, and D. Estrin, "An energy-efficient MAC protocol for wireless sensor network,"In: Proceedings of the $21^{s t}$ International Annual Joint Conference of the IEEE Computer and Communications Societies(INFOCOM'02), 2002, pp. 1567-1576.

[6] J. Deng, Y.S. Han, W.B. Heinzelman, and P.K. Varshney, "Scheduling sleeping nodes in high density cluster-based sensor network," In: ACM/Kluwer Mobile Network and Applications (MONET) Special Issue on Energy Constraints and Lifetime Performance in Wireless Sensor Network, 2005, pp. 825-835.

[7] J. Deng, Y.S. Han, W.B. Heinzelman, and P.K. Varshney, "Balanced-Energy sleep scheduling scheme for high density cluster-based sensor network," Elsevier Computer Communications Journal (Special Issue on ASWN'04), vol. 28, no. 14, pp. 1631$1642,2005$.

[8] Q. Cao, T. Abdelzaher, T. He, and J. Stankovic, "Towards optimal sleep scheduling in sensor network for rare event detection," In: Proceedings of the $4^{\text {th }}$ International Symposium on Information Processing in Sensor Network (IPSN'05), 2005.

[9] G. Yu, and T. He, "Dynamic switching-based data forwarding for low-duty-cycle wireless sensor network,' In: Mobile Computing, IEEE Transactions on, 2011.

[10] Y. Kim, R. Govindan, B. Karp, and S. Shenker, "Geographic routing made practical," In: Proceedings of the $2^{\text {nd }}$ conference on Symposium on Networked Systems Design \& Implementation NSDI'05, 2005.

[11] H. Hao, Y. Jihoon, Z. Ziguo, K. Songmin, and T. He, "PSR: Practical synchronous rendezvous in low-duty-cycle wireless network," In: Proceeding of IEEE Infocom, 2013, pp. 2661-2669. 